\title{
Yang-Mills gauge theories from simple fermionic lattice models
}

\author{
Paolo Maraner ${ }^{\mathrm{a}}$ and Jiannis K. Pachos ${ }^{\mathrm{b}}$

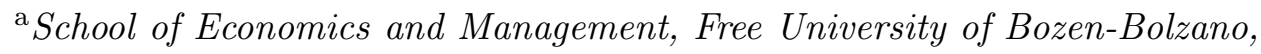 \\ via Sernesi 1, 39100 Bolzano, Italy \\ ${ }^{\mathrm{b}}$ School of Physics and Astronomy, University of Leeds, Leeds LS2 9JT, UK
}

\begin{abstract}
A doublet of three-dimensional Dirac fermions can effectively describe the low energy spectrum of a fermionic cubic lattice. We employ this fermion doubling to encode a non-Abelian $S U(2)$ charge in the fundamental representation. We explicitly demonstrate that suitable distortion of the tunnelling couplings can introduce a scalar and a Yang-Mills field in the effective low energy description, both coupled to the Dirac fermions. The simplicity of the model suggests its physical implementation with ultra-cold atoms or molecules.
\end{abstract}

Key words: Yang-Mills, fermionic lattices.

\section{Introduction}

Recently, much interest has been focused on fermionic lattice models that can simulate one or two-dimensional exotic phenomena such as fractionalization of charge or anyonic statistics. Polyacetylene is a one-dimensional example, which gave a simple and rigorous theoretical model for the charge fractionalization [1] that enjoyed experimental verification [2]. In two-dimensions graphene, a mono-atomic layer of graphite, has a low energy description given by a twodimensional Dirac equation [3]. It has been shown that under certain coupling distortions of the underlining lattice it can support axial gauge theories that lead to the fractionalization of charge [4,5,6,7]. In three or four dimensions the properties of these lattices and their coupling to external non-Abelian gauge fields are well understood, for example, in the context of staggered fermion lattice gauge theories [10,11,12]. How non-Abelian gauge fields can effectively arise in the continuum limit without employing them already in the lattice picture remained up to now an open question [5,8,9]. 
In this letter, we systematically study the case of three spatial dimensions. We employ a cubic lattice model where fermions are tunnelling from site to site with specific transition couplings. It has been shown [13] that the low energy spectrum of this model is effectively described by three-dimensional Dirac spinors. Here, we demonstrate that suitable distortions of the transition couplings and of the local potentials give rise to additional triplet scalar and Yang-Mills fields, both of them coupled to the Dirac field. Importantly, the presented distortions do not originate from non-Abelian fields in the lattice picture. The interest in such structures is diverse. The simplicity of these lattice models could inspire physical systems such as optical lattices or multilayer graphene that can simulate Dirac fermions coupled to dynamical YangMills gauge fields. Such an endeavor could establish a bridge between the lattice gauge theory and the experimental condensed matter communities. Moreover, it is of interest to see if the standard model can emerge as an effective theory of a simple underlined lattice structure [9]. Such a model could generate Abelian and non-Abelian gauge potentials as well as Higgs scalar fields that emerge without a symmetry breaking due to a simple quantum ordered in its ground state.

\section{The three dimensional case}

\subsection{The fermionic lattice model and the Dirac equation}

For concreteness we consider the three-dimensional Hamiltonian

$$
D \Psi=\left(\mathbb{1} \otimes \boldsymbol{\gamma} \cdot \mathbf{p}-T^{a} \otimes \boldsymbol{\gamma} \cdot \mathbf{A}_{a}+T^{a} \otimes \mathbb{1} \Phi_{a}\right) \Psi
$$

where summations are assumed over repeated indices. The gauge field is taken to be in the fundamental representation of $S U(2)$, i.e. $T^{a}=\frac{\sigma_{a}}{2}$ [14]. The four spinor, $\Psi_{i}^{(n)}$, is indexed by $i=1, \ldots, 4$, while $n=1,2$ runs trough the color components. The index $a=1,2,3$ runs through the gauge field components

and $\left\{\gamma_{\mu}, \gamma_{\nu}\right\}=2 \delta_{\mu \nu}$, where $\mu, \nu$ run through the space components $x, y$ and $z$. By choice of gauge the three-dimensional gauge vector $\mathbf{A}_{a}$ and the triplet scalar field $\Phi_{a}$ are taken to be real.

Our starting point is a cubic lattice where fermions tunnel from one site to the neighboring one according to the simple quadratic Hamiltonian

$$
\hat{H}=-\sum_{\langle\mathbf{i}, \mathbf{j}\rangle} \psi_{\mathbf{i}}^{\dagger} X_{\mathbf{i j}} \psi_{\mathbf{j}}+\text { H.c. }
$$




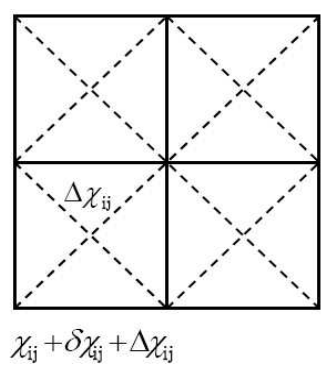

Fig. 1. A two-dimensional section of the three-dimensional cubic lattice. Fermions, $\psi_{\mathbf{i}}$, tunnel from site $\mathbf{i}$ to site $\mathbf{j}$ along the links of the lattice with couplings $X_{\mathbf{i j}}=\chi_{\mathbf{i j}}+\delta \chi_{\mathbf{i j}}+\Delta \chi_{\mathbf{i j}}$. Here $\chi_{\mathbf{i j}}$ and $\delta \chi_{\mathbf{i j}}$ couple nearest neighboring sites, $\mathbf{i}$ and $\mathbf{j}$, that lie only along the same link of the cubic lattice, while $\Delta \chi_{\mathbf{i j}}$ couples diagonal sites as well.

The tunnelling couplings are given by

$$
X_{\mathbf{i j}}=\chi_{\mathbf{i j}}+\delta \chi_{\mathbf{i j}}+\Delta \chi_{\mathbf{i j}}
$$

where $\chi_{\mathbf{i j}}$ correspond to a uniform pattern, while $\delta \chi_{\mathbf{i j}}$ and $\Delta \chi_{\mathbf{i j}}$ correspond to coupling distortions that could have a slow spatial variation. While $\chi_{\mathbf{i j}}$ and $\delta \chi_{\mathbf{i j}}$ connect nearest neighbor sites that lie only along the links of the cubic lattice, the couplings $\Delta \chi_{\mathbf{i j}}$ can also generate site energy shifts or couple sites along diagonals, as seen in Fig. 1. As we shall see the couplings $\chi_{\mathbf{i j}}$ are chosen so that the low energy description of $\hat{H}$ is given by a Dirac equation. There are three independent components of $\delta \chi_{\mathbf{i j}}$, along the different directions of the lattice, that can give rise to a triplet scalar field, while nine independent components of $\Delta \chi_{\mathbf{i j}}$ can be identified that can give rise to the $S U(2)$ non-Abelian gauge potential.

Let us first consider the effect of the uniform couplings $\chi_{\mathbf{i j}}$ that give rise to the Kogut-Susskind (or staggered) fermions [13]. Their particular values that actually minimize the energy of Hamiltonian (2) are given by

$$
\chi_{\mathbf{i}, \mathbf{i}+\hat{\mathbf{x}}}=-\frac{i}{2}, \chi_{\mathbf{i}, \mathbf{i}+\hat{\mathbf{y}}}=-\frac{i}{2}(-1)^{i_{x}}, \chi_{\mathbf{i}, \mathbf{i}+\hat{\mathbf{z}}}=-\frac{i}{2}(-1)^{i_{x}+i_{y}} .
$$

This configuration corresponds to a $\pi$-flux going through each square plaquette of the lattice. As the couplings, $\chi_{\mathbf{i j}}$, have alternating signs along the $x$ and $y$ directions, while they are homogeneous along the $z$ direction, the resulting Brillouin zone, $B$, is given by $k_{x}, k_{y} \in[-\pi / 2, \pi / 2]$ and $k_{z} \in[-\pi, \pi]$. In momentum space the Hamiltonian kernel, $H$, is given by

$$
H=\mathbb{1} \otimes \sigma_{3} \sin k_{x}+\sigma_{3} \otimes \sigma_{1} \sin k_{y}+\sigma_{1} \otimes \sigma_{1} \sin k_{z}
$$

in the basis $\left(\mathbf{k}, \mathbf{k}+\mathbf{Q}_{\mathbf{x}}, \mathbf{k}+\mathbf{Q}_{\mathbf{y}}, \mathbf{k}+\mathbf{Q}_{\mathbf{x}}+\mathbf{Q}_{\mathbf{y}}\right)$, where $\mathbf{Q}_{\mathbf{x}}=(\pi, 0,0)$ and $\mathbf{Q}_{\mathbf{y}}=$ $(0, \pi, 0)$. The corresponding eigenvalues of the energy are given by

$$
E_{ \pm}= \pm \sqrt{\sin ^{2} k_{x}+\sin ^{2} k_{y}+\sin ^{2} k_{z}}
$$


Within the Brillouin zone, $B$, there are two isolated Fermi points given by $\mathbf{K}_{+}=(0,0,0)$ and $\mathbf{K}_{-}=(0,0, \pi)$ that set the energy, $E_{ \pm}$, to zero. Focusing on the low energy regime of the Hamiltonian at half filling we expand (4) around $\mathbf{K}_{+}$and $\mathbf{K}_{-}$. For $\mathbf{k}=\mathbf{K}_{ \pm}+\mathbf{p}$ we have

$$
H_{ \pm}=\mathbb{1} \otimes \sigma_{3} p_{x}+\sigma_{3} \otimes \sigma_{1} p_{y} \pm \sigma_{1} \otimes \sigma_{1} p_{z}
$$

where $|\mathbf{p}|$ is taken to be small. We can now define $\gamma_{x}=\mathbb{1} \otimes \sigma_{3}, \gamma_{y}=\sigma_{3} \otimes \sigma_{1}$ and $\gamma_{z}=\sigma_{1} \otimes \sigma_{1}$ so that $H_{+}=\boldsymbol{\gamma} \cdot \mathbf{p}$. The corresponding spinors are given by $\Psi_{ \pm}(\mathbf{p})^{T}=\left(\psi_{ \pm, \mathbf{p}}, \psi_{ \pm, \mathbf{p}+\mathbf{Q}_{x}}, \psi_{ \pm, \mathbf{p}+\mathbf{Q}_{y}}, \psi_{ \pm, \mathbf{p}+\mathbf{Q}_{x}+\mathbf{Q}_{y}}\right)$ where $\psi_{ \pm, \mathbf{p}}=\psi\left(\mathbf{K}_{ \pm}+\mathbf{p}\right)$. Since $\left(\sigma_{3} \otimes \mathbb{1}\right) H_{-}\left(\sigma_{3} \otimes \mathbb{1}\right)=H_{+}$it is easy to show that for $U=\operatorname{diag}(\mathbb{1} \otimes$ $\left.\mathbb{1}, \sigma_{3} \otimes \mathbb{1}\right)$ it is

$$
U\left(\begin{array}{cc}
H_{+} & 0 \\
0 & H_{-}
\end{array}\right) U^{\dagger}=\mathbb{1} \otimes \boldsymbol{\gamma} \cdot \mathbf{p} .
$$

In this way one can obtain two identical copies of the free three-dimensional Dirac equation. The spinor, $\Psi$, of eqn. (1) is given by $\Psi_{i}^{(1)}=U \Psi_{+i}$ and $\Psi_{i}^{(2)}=U \Psi_{-i}$, where $i=1, \ldots, 4$ enumerates the four components of the spinors.

\subsection{The scalar field}

In order to obtain the scalar field of Hamiltonian (11) we choose the coupling distortions to be of the form

$$
\delta \chi_{\mathbf{i}, \mathbf{i}+\hat{\mathbf{y}}}=-\frac{\Phi}{4} e^{-i \mathbf{K}_{-} \cdot \mathbf{i}} e^{i \mathbf{K}_{+} \cdot\left(\mathbf{i}+\mathbf{s}_{y}\right)}, \delta \chi_{\mathbf{i}, \mathbf{i}+\hat{\mathbf{z}}}=-\frac{\Phi_{3}}{8},
$$

where $\Phi=\Phi_{1}-i \Phi_{2}$ with all $\Phi_{a}$ real. The $\mathbf{i}$ vector indicates the position of the corresponding site and the $\mathbf{s}_{a}$ vector indicates its two nearest neighbors along the direction $a$. The components of the scalar field can have a variation in space, which is slow compared to the lattice spacing. As $\delta \chi_{\mathbf{i}, \mathbf{i}+\hat{\mathbf{x}}}=0$ we have that $\delta H^{(x)}=0$. For the distortion $\delta \chi_{\mathbf{i}, \mathbf{i}+\hat{\mathbf{y}}}$ and for momenta close to the Fermi points we obtain the additional Hamiltonian term

$$
\delta H^{(y)}=\frac{\Phi_{1} \sigma_{1}+\Phi_{2} \sigma_{2}}{2} \otimes \sigma_{3} \otimes \mathbb{1} .
$$

The distortion in the $z$ direction, $\delta \chi_{\mathbf{i}, \mathbf{i}+\hat{\mathbf{z}}}$, gives the Hamiltonian contribution

$$
\delta H^{(z)}=\frac{\Phi_{3}}{2} \sigma_{3} \otimes \mathbb{1} \otimes \mathbb{1} .
$$

Finally, the conjugation $U\left(\delta H^{(y)}+\delta H^{(z)}\right) U^{\dagger}$ results to the triplet scalar field term, $T^{a} \otimes \mathbb{1} \Phi_{a}$, of Hamiltonian (1). 


\subsection{The Yang-Mills field}

The gauge field can originate from the $\Delta \chi_{\mathbf{i j}}$ distortion. The non-zero couplings are given by

$$
\begin{aligned}
& \Delta \chi_{\mathbf{i}, \mathbf{i}+\hat{\mathbf{y}}}=(-1)^{i_{x}+i_{y}} \frac{A^{z}}{4} e^{-\mathbf{K}_{-} \cdot \mathbf{i}} e^{i \mathbf{K}_{+} \cdot \mathbf{i}}, \\
& \Delta \chi_{\mathbf{i}, \mathbf{i}}=-(-1)^{i_{x}+i_{y}} \frac{A_{3}^{z}}{4}-(-1)^{i_{x}} \frac{A^{y}}{4} e^{-i \mathbf{K}_{-} \cdot \mathbf{i}} e^{i \mathbf{K}_{+} \cdot \mathbf{i}}, \\
& \Delta \chi_{\mathbf{i}, \mathbf{i}+\hat{\mathbf{x}}+\hat{\mathbf{y}}}=-\frac{A^{x}}{8} e^{-i \mathbf{K}_{-} \cdot \mathbf{i}} e^{i \mathbf{K}_{+} \cdot \mathbf{i}}, \\
& \Delta \chi_{\mathbf{i}, \mathbf{i}+\hat{\mathbf{x}}+\hat{\mathbf{z}}}=-\frac{A_{3}^{x}}{16}, \Delta \chi_{\mathbf{i}, \mathbf{i}+\hat{\mathbf{y}}+\hat{\mathbf{z}}}=-(-1)^{i_{x}} \frac{A_{3}^{y}}{16}
\end{aligned}
$$

where $A^{\mu}=A_{1}^{\mu}-i A_{2}^{\mu}$ with all $A_{a}^{\mu}$ real and slowly varying with respect to the lattice spacing. These distortions of the tunnelling couplings provide the nine components of the non-Abelian gauge potential. Indeed, $\Delta \chi_{\mathbf{i}, \mathbf{i}+\hat{\mathbf{y}}}$ gives rise to

$$
\Delta H^{(y)}=-\frac{A_{1}^{z} \sigma_{2}-A_{2}^{z} \sigma_{1}}{2} \otimes \sigma_{2} \otimes \sigma_{1} .
$$

From $\Delta \chi_{\mathbf{i}, \mathbf{i}}$, which is a distortion of the local potential of site $\mathbf{i}$, we obtain

$$
\Delta H^{(\mathbf{0})}=-\frac{A_{3}^{z}}{2} \mathbb{1} \otimes \sigma_{1} \otimes \sigma_{1}-\frac{A_{1}^{y} \sigma_{1}+A_{2}^{y} \sigma_{2}}{2} \otimes \mathbb{1} \otimes \sigma_{1} .
$$

From $\Delta \chi_{\mathbf{i}, \mathbf{i}+\hat{\mathbf{x}}+\hat{\mathbf{y}}}$ we obtain the additional Hamiltonian term

$$
\Delta H^{(\hat{\mathbf{x}}+\hat{\mathbf{y}})}=-\frac{A_{1}^{x} \sigma_{1}+A_{2}^{x} \sigma_{2}}{2} \otimes \sigma_{3} \otimes \sigma_{3},
$$

while from $\Delta \chi_{\mathbf{i}, \mathbf{i}+\hat{\mathbf{x}}+\hat{\mathbf{z}}}$ we obtain

$$
\Delta H^{(\hat{\mathbf{x}}+\hat{\mathbf{z}})}=-\frac{A_{3}^{x}}{2} \sigma_{3} \otimes \mathbb{1} \otimes \sigma_{3} .
$$

Finally, from $\Delta \chi_{\mathbf{i}, \mathbf{i}+\hat{\mathbf{y}}+\hat{\mathbf{z}}}$ we obtain

$$
\Delta H^{(\hat{\mathbf{y}}+\hat{\mathbf{z}})}=-\frac{A_{3}^{y}}{2} \sigma_{3} \otimes \sigma_{3} \otimes \sigma_{1} .
$$

After conjugating by $U$ we have that $U\left(\Delta H^{(y)}+\Delta H^{(\mathbf{0})}+\Delta H^{(\hat{\mathbf{x}}+\hat{\mathbf{y}})}+\Delta H^{(\hat{\mathbf{x}}+\hat{\mathbf{z}})}+\right.$ $\left.\Delta H^{(\hat{\mathbf{y}}+\hat{\mathbf{z}})}\right) U^{\dagger}=-T^{a} \otimes \boldsymbol{\gamma} \cdot \mathbf{A}_{a}$. This shows that the $\Delta \chi_{\mathbf{i j}}$ coupling distortions followed by the $U$ conjugation give rise to the gauge potential term of Hamiltonian (11). Thus, by appropriate distortions of the tunnelling couplings of the fermionic lattice model one can generate a non-Abelian gauge and a scalar field coupled to a Dirac field. Note, that, while these distortions may have a 
complex value the total additional plaquette flux they introduce is zero. Thus, the initial lattice Hamiltonian (2) is time symmetric.

\subsection{Yang-Mills monopoles and the index theorem}

In our construction the gauge and scalar field components can take arbitrary configurations. As an application, we would like to simulate Yang-Mills monopoles in the low energy behavior of the presented fermionic lattice model by appropriately choosing the coupling distortions, $\delta \chi_{\mathbf{i j}}$ and $\Delta \chi_{\mathbf{i j}}$. The field configurations, induced by a single monopole positioned at the origin with unit charge, are given by

$$
\Phi_{a}=\hat{r}_{a} \Phi(r), A_{a}^{\mu}=\epsilon^{a \mu \nu} \hat{r}_{\nu} A(r) .
$$

Both $\Phi(r)$ and $A(r)$ are taken to vanish at $r=0$, while for large $r$ the scalar field tends to its vacuum value, $\Phi \underset{r \rightarrow \infty}{\longrightarrow} m$, and the gauge field behaves as $A \underset{r \rightarrow \infty}{\longrightarrow}-1 / r$, where the approach to their asymptotic behavior is exponential [15]. Substituting these configurations into Hamiltonian (1) one obtains

$$
D \Psi=\left[\mathbb{1} \otimes \boldsymbol{\gamma} \cdot \mathbf{p}-\frac{\sigma_{a}}{2} \otimes(\boldsymbol{\gamma} \times \hat{\mathbf{r}})_{a} A+\frac{\sigma_{a}}{2} \otimes \mathbb{1} \hat{r}_{a} \Phi\right] \Psi
$$

which describes the Yang-Mills monopole coupled to a Fermi field.

The appearance of zero fermionic modes is the most striking characteristic of such non-trivial topological configurations [16]. One can employ the index theorem for open spaces to estimate the number of these zero energy modes [17,18]. The index of an operator of the form

$$
D=\left(\begin{array}{cc}
0 & L \\
L^{\dagger} & 0
\end{array}\right)
$$

is defined by $\operatorname{index}(D)=k_{+}-k_{-}$, where $k_{+}\left(k_{-}\right)$is the number of zero modes of $L\left(L^{\dagger}\right)$. Its absolute value gives a lower bound to the total number of zero modes, $k_{+}+k_{-}$, of $D$. It is possible to bring Hamiltonian (10) in the form (11) by conjugating it with $\mathbb{1} \otimes\left[S\left(\mathbb{1} \otimes e^{i \sigma_{1} \pi / 4}\right)\right]$, where $S\left(\sigma_{a} \otimes \sigma_{b}\right)=\sigma_{b} \otimes \sigma_{a}$ for all the Pauli matrices including $\sigma_{0}=\mathbb{1}$. Then the index of Hamiltonian (10) is given by

$$
\operatorname{index}(D)=-\frac{1}{8 \pi} \int_{S_{\infty}^{2}} d S^{\mu} \epsilon^{\mu \alpha \beta} \epsilon^{a b c} \frac{\Phi_{c} \partial_{\alpha} \Phi_{a} \partial_{\beta} \Phi_{b}}{|\Phi|^{3}},
$$

which corresponds to the degree of $\Phi_{a}$ if it is considered as a mapping from $S_{\infty}^{2}$ to $S_{m}^{2}$. For the single Yang-Mills monopole given in (9) with charge 1 we have index $(D)=1$ indicating that the system has (at least) one zero mode. 
Similarly, it is possible to simulate higher charge monopoles, the explicit forms of which are given in [19].

It has been demonstrated [20] by analytic calculations that the monopole configuration (9) possesses a unique normalized, isolated and non-degenerate zero-energy mode. Its wave function is given by $\Psi_{E=0}^{(n)}=\left(\phi^{(n)}, 0\right)^{T}$, where $\phi^{(n)}$ is a two component spinor

$$
\phi_{i}^{(n)}(r)=N i \exp \left(-\int_{0}^{r} d r^{\prime}\left[\frac{1}{2} \Phi\left(r^{\prime}\right)+A\left(r^{\prime}\right)\right]\right)\left(\delta_{1 i} \delta_{2 n}-\delta_{2 i} \delta_{1 n}\right)
$$

with $i=1,2$ and $N$ a real normalization constant. This solution corresponds to a wave function that is well localized around the position of the monopole, $r=0$. The presence of a single zero mode in the spectrum of the Dirac operator, together with the symmetry under fermi-number conjugation, $\Psi^{(n) c} \equiv \sigma_{3} \otimes \sigma_{2}\left(\sigma_{2}\right)_{n m} \Psi^{(m) *}$, of Hamiltonian (10) signals the fractionalization of the fermion number [21]. Thus, the presence of a classical topological defect in the configurations of the Yang-Mills and scalar fields causes the fermionic field to take fractional quantum numbers. This can be detected in the lattice fermion system by comparing the fermion densities with and without the monopole. A fermion density difference that corresponds to the localized wave function (13) provides a clear signature of the charged fractionalization.

\section{The two dimensional case}

For completeness we would like to present the generation of the Yang-Mills field in the two-dimensional case. For that consider a square lattice with tunnelling fermions governed by a similar Hamiltonian as in (1). The transition couplings are given by $X_{\mathbf{i j}}=\chi_{\mathbf{i} \mathbf{j}}+\Delta \chi_{\mathbf{i} \mathbf{j}}$, with

$$
\chi_{\mathbf{i}, \mathbf{i}+\hat{\mathbf{x}}}=-\frac{i}{2}, \chi_{\mathbf{i}, \mathbf{i}+\hat{\mathbf{y}}}=-\frac{i}{2}(-1)^{i_{x}}
$$

and

$$
\begin{aligned}
& \Delta \chi_{\mathbf{i}, \mathbf{i}}=(-1)^{i_{x}} \frac{A_{3}^{y}}{4}+\frac{A^{x}}{2} e^{-i\left(\mathbf{K}_{+}-\mathbf{K}_{-}\right) \cdot \mathbf{i}}, \\
& \Delta \chi_{\mathbf{i}, \mathbf{i}+\hat{\mathbf{x}}+\hat{\mathbf{y}}}=\frac{A_{3}^{x}}{4} \\
& \Delta \chi_{\mathbf{i}, \mathbf{i}+\hat{\mathbf{x}}}=(-1)^{i_{x}} \frac{A^{y}}{2} e^{-i\left(\mathbf{K}_{+}-\mathbf{K}_{-}\right) \cdot \mathbf{i}},
\end{aligned}
$$

where $A^{\mu}=A_{1}^{\mu}-i A_{2}^{\mu}$, the Fermi points are $\mathbf{K}_{+}=(0,0)$ and $\mathbf{K}_{-}=(0, \pi)$ and the Brillouin zone, $B$, is given by $k_{x} \in[-\pi / 2, \pi / 2]$ and $k_{y} \in[-\pi, \pi]$. The 
homogeneous tunnelling couplings $\chi_{\mathbf{i j}}$ give rise to a pair of two-dimensional Dirac equation corresponding to the two Fermi points. Under the conjugation by $U=\operatorname{diag}\left(\mathbb{1}, \sigma_{3}\right)$ the composite Hamiltonian becomes $\mathbb{1} \otimes \boldsymbol{\gamma} \cdot \mathbf{p}$, where $\gamma=\left(\sigma_{3}, \sigma_{1}\right)$. The distortion $\Delta \chi_{\mathbf{i}, \mathbf{i}}$ of the local site energies gives rise to

$$
\delta H^{(\mathbf{0})}=-\frac{A_{3}^{y}}{2} \mathbb{1} \otimes \sigma_{1}-\frac{A_{1}^{x} \sigma_{1}+A_{2}^{x} \sigma_{2}}{2} \sigma_{1} \otimes \mathbb{1} .
$$

From the distortion $\Delta \chi_{\mathbf{i}, \mathbf{i}+\hat{\mathbf{x}}+\hat{\mathbf{y}}}$ we obtain

$$
\Delta H^{(\hat{\mathbf{x}}+\hat{\mathbf{y}})}=-\frac{A_{3}^{x}}{2} \sigma_{3} \otimes \sigma_{3}
$$

while from $\Delta \chi_{\mathbf{i}, \mathbf{i}+\hat{\mathbf{x}}}$ we have

$$
\Delta H^{(\hat{\mathbf{x}})}=-\frac{A_{1}^{y} \sigma_{2}-A_{2}^{y} \sigma_{1}}{2} \otimes \sigma_{2}
$$

It is easy now to show that under conjugation by $U$ the overall Hamiltonian produces the two dimensional Dirac operator coupled to an $S U(2)$ Yang-Mills field $D \Psi=\left(\mathbb{1} \otimes \boldsymbol{\gamma} \cdot \mathbf{p}-T^{a} \otimes \boldsymbol{\gamma} \cdot \mathbf{A}_{a}\right) \Psi$.

It is an exciting possibility that such a lattice Hamiltonian could be generated with optical lattices and ultra-cold atom or molecule technology [22]. In particular, the generation of the $\pi$-flux through the square plaquettes of optical lattices is possible by imprinting phases with laser assisted tunnelling processes [23]. The presented method for generating Yang-Mills fields has the advantage that it does not employ the internal states of the atoms that demand additional laser fields [24] and, thus, it requires a significantly simpler experimental setting for its physical realization. For example, the distortion of the tunnelling couplings is expected to appear naturally in optical lattice systems due to amplitude variations of the corresponding laser fields. Alternatively, controlled spatially profiles of laser fields can be easily produced in the laboratory without increasing the complexity of the experiment. Applications of non-Abelian fields that facilitate the robust simulation of the quantum Hall effect with ultra-cold atoms have been reported in [25].

\section{Conclusions}

In conclusion, we considered a fermionic system with discretized three spatial dimensions described by the Lagrangian

$$
\mathcal{L}=\sum_{\mathbf{i}} \psi_{\mathbf{i}}^{\dagger} i\left(\partial_{t}+i A^{0}\right) \psi_{\mathbf{i}}+\left(\sum_{\langle\mathbf{i} \mathbf{j}} \psi_{\mathbf{i}}^{\dagger} X_{\mathbf{i j}} \psi_{\mathbf{j}}+\text { H.c. }\right)
$$


where time is considered to be a continuous variable. By a particular choice of the transition couplings of the fermions we demonstrated that the low energy limit of this system is faithfully described by Dirac fermions coupled to a triplet scalar field, also known as a Higgs field, and a Yang-Mills $S U(2)$ nonAbelian gauge field. These fields can be chosen to encode Yang-Mills-Higgs monopole configurations. In particular, a single monopole with charge 1 can give rise to an isolated zero-mode. We demonstrated that such a setting gives rise to fractionalization of charge in three dimensions. Moreover, we derived the analytic relation between the lattice fermions wave function and the Dirac ones, thus making it possible to detect the topological effect of the effective gauge theory by probing the behavior of the constituent lattice particles. It is intriguing to see if such systems in two or three dimensions can be simulated in the laboratory.

The presented simulation opens up numerous possibilities. It would be interesting to generate kinetic terms for the Yang-Mills field and augment it to an independent dynamical entity in a quantum field theory. In this context the study of the full symmetry group of the lattice and the emergence of the correct massless spectrum in the quantum continuum limit is highly desirable. Alternatively, a proof of stability of the quantum order of these non-Abelian systems as well as the encoding of chiral fermions would bring this effort closer into a full blown simulation of the standard model.

\section{Acknowledgments}

We thank Roman Jackiw and So-Young Pi for inspiring conversations throughout this project. We would like to acknowledge the hospitality of the Aspen Center for Physics. This work was supported by the EU grants EMALI and SCALA, EPSRC and the Royal Society.

\section{References}

[1] M. J. Rice and E. J. Mele, Phys. Rev. Lett. 49, 1455 (1982); R. Jackiw and G. Semenoff, Phys. Rev. Lett. 50, 439 (1983); R. Jackiw and J. R. Schrieffer, Nucl. Phys. B190, 253 (1981).

[2] S. A. Kivelson, Synthetic Metals 125, 99 (2002) and references therein.

[3] P. R. Wallace, Phys. Rev. 71622 (1947).

[4] C.-Y. Hou, C. Chamon and C. Mudry, Phys. Rev. Lett. 98, 186809 (2007). 
[5] R. Jackiw, and S.-Y. Pi, Phys. Rev. Lett. 98, 266402 (2007); C. Chamon, C.-Y. Hou, R. Jackiw, C. Mudry, S.-Y. Pi, and A. P. Schnyder, Phys. Rev. Lett. 100, 110405 (2008).

[6] B. Seradjeh, C. Weeks, M. Franz, Phys. Rev. B 77, 033104 (2008).

[7] J. K. Pachos, M. Stone, and K. Temme, Phys. Rev. Lett. 100, 156806 (2008).

[8] G. E. Volovik, JETP Letters 43, 551 (1986); "The Universe in a Helium Droplet", Clarendon Press, Oxford (2003).

[9] X.-G. Wen, Phys. Rev. Lett. 88, 011602 (2001).

[10] M. F. Golterman and J. Smit, Nucl. Phys. B 245, 61 (1984).

[11] H. Kluberg-Stern, A. Morel, O. Napoly and B. Petersson, Nucl. Phys. B 220, 447 (1983).

[12] P. Mitra and P. Weisz, Phys. Lett. B 126, 355 (1983).

[13] J. B. Kogut, and L. Susskind, Phys. Rev. D 11, 395 (1975); L. Susskind, Phys. Rev. D 16, 3031 (1977).

[14] Here $\sigma_{a}, a=1,2,3$ are the Pauli matrices and $\sigma_{0}=\mathbb{1}$ is the two dimensional identity matrix.

[15] G. 't Hooft, Nucl. Phys. D79, 276 (1974); A. M. Polyakov, JETP Lett. 20, 194 (1974).

[16] E. Follana, A. Hart, C. T. H. Davies and Q. Mason, Phys. Rev. D 72, 054501 (2005).

[17] C. Callias, Commun. Math. Phys. 62, 213 (1978).

[18] R. Bott and R. Seeley, Commun. Math. Phys. 62, 235 (1978).

[19] R. S. Ward, Commun. Math. Phys. 79, 317-325 (1981); M. K. Prasad, Commun. Math. Phys. 80, 137-149 (1981).

[20] R. Jackiw and C. Rebbi, Phys. Rev. D 13, 3398 (1976).

[21] R. Jackiw, math-ph/0503039 (2005).

[22] G. Pupillo, A. Micheli, H. P. Büchler, and P. Zoller, Chapter of the book: Cold molecules: Creation and applications, Taylor \& Francis (2008); H. P. Büchler, E. Demler, M. Lukin, A. Micheli, N. Prokofév, G. Pupillo, and P. Zoller, Phys. Rev. Lett. 98, 060404 (2007).

[23] D. Jaksch and P. Zoller, New J. Phys. 5, 56 (2003).

[24] K. Osterloh, M. Baig, L. Santos, P. Zoller, and M. Lewenstein, Phys. Rev. Lett. 95, 010403 (2005).

[25] N. Goldman, A. Kubasiak, P. Gaspard, and M. Lewenstein, arXiv:0712.2571 (2007). 